require lower doses of antipsychotic medication and benzodiazepines than young men. Gender is considered to be an important variable in the processes of absorption, distribution, metabolism and excretion of drugs (Fletcher et al, 1994). Gastric acid secretion, gastro-intestinal blood flow, proportions of muscular and adipose tissue, amount of drug-binding proteins, gender-specific cytochrome p450 isozymes, physiological and hormonal changes during the menstrual cycle, and renal blood flow have been highlighted by Fletcher $e t$ al as factors that may contribute to gender-related differences in pharmacokinetics. Cytochrome p450, the primary oxidative pathway of drug metabolism and a major site of drug, drug-diet and drug-disease/ condition interactions (Rogers, 1994), is increasingly recognised as important in psychopharmacology (von Moltke et al, 1994). Isozymes of cytochrome p450 that are specific to females are known to exist and are reported to have significant influence on side-chain oxidation, especially on C-oxidation (Fletcher et al, 1994). Gender influences on psychopharmacology are therefore important. Future research should focus not only on the pathophysiology of conditions restricted to or more relevant in women, but also aim to provide more gender-specific information on drug disposition and clinical effect.

Crnousx, H. \& Welch, S. (1996) Women and psychiatry. British Journal of Psychiatry, 169, 6-9.

Fletcher, C. V., Acosta, E. P. \& StryxowsKi, J. M. (1994) Gender differences in human pharmacokinetics and pharmacodynamics. Journal of Adolescent Health, 15, 619-629.

RoGerRs, A. S. (1994) The role of cytochrome P450 in developmental pharmacology. Journal of Adolescent Health, 15, 635-640.

von MoltKe, L. L., Greenblatt, D. J., Harmatz, J. S., et al (1994) Cytochromes in psychopharmacology. Journal of Clinical Psychopharmacology, 14, 1-4.

YonkKRrs, K. A., KANDO, J., Kole, J. O., et al (1992) Gender differences in pharmacodynamics and pharmacokinetics of psychotropic medication. American Journal of Psychiatry, 149, 587-595.

Shaftesbury Clinic

61 Glenburnie Road

London SE17 7DJ

Department of General Psychiatry

S. R. JACOBSON

St George's Medical School

London SW17 ORE

\section{Melancholia and response to ECT}

SIR: I was interested to read the study by Hickie et al (1996) but am keen to offer an alternative interpretation of their results. They found that degree of psychomotor retardation and presence of psychotic ideas better predict response to ECT than the initial severity of depression as measured by the Hamilton Rating Scale for Depression (HRSD). They conclude that this is supportive of the validity of melancholia, a postulated sub-type of depression.

The deficiencies of the HRSD have been well documented (Gibbons et al, 1993), especially for a group of predominantly elderly patients with a wide age-range and high rates of concurrent medical problems (Snaith, 1993), such as Hickie et als sample. It is therefore quite likely that psychomotor retardation and psychotic ideas were simply much better markers of severe depressive illness in this study than the HRSD score was. Therefore, a person with psychomotor retardation and psychotic ideas but with a lower HRSD score is likely to be more depressed than one with a high score but lacking these major symptoms. This is borne out by Hickie et $a l s$ findings of stronger correlations between the initial Global Assessment of Functioning (GAF) and psychotic symptoms and retardation than between the initial HRSD and GAF scores. Viewed in this light, Hickie et al's findings should be interpreted as showing that the likelihood of response to ECT depends upon the initial severity of illness and that severe illness is best characterised by poor global functioning and the presence of psychotic thought or psychomotor retardation. There is no need to postulate a sub-type of depression.

Gibbons, R. D., Clark, D. C. \& Kupfer, D. J. (1993) Exactly what does the Hamilton depression rating scale measure? Journal of Psychiatric Research, 27, 259-273.

Hickie, I., Mason, C., PArker, G., et al (1996) Prediction of ECT response: validation of a refined sign-based (CORE) system for defining melancholia. British Journal of Psychiatry, 169, 68-74.

SNArTH, P. (1993) What do depression rating scales measure? British Journal of Psychiatry, 163, 293-298.

Hellesdon Hospital

J. M. G. Winliams

Drayton High Road

Norwich NR6 SBE

\section{Urine screening for drugs and trazodone}

SIR: I write to draw the reader's attention to the interesting but spurious appearance of positive amphetamine results in the presence of trazodone on routine drug screening with polyclonal antibodies such as EMIT-1 (originally produced by SYVA). Reporting of amphetamines, therefore, on routine drug screening while a patient is on trazodone may be spurious. A confirmatory test 
should be requested to clarify whether or not amphetamines are present in the urine.

MOORE, F. M. L., JARVE, D. R. \& SMPSON, D. (1996) Comparison
of polyclonal and monoclonal tests for routine screening of urine for amphetamines. Annals of Clinical Biochemistry, 33, 78-81.

\section{Loganlea Centre}

76 John Street, Penicuik

Midlothian EH26 8NF

\section{Costing community care}

SIR: We were concerned to read the interesting article by Salize \& Rössler (1996). There is little knowledge of the full costs of community care. They study acute psychiatric patients who are well enough to be discharged into independent living, who thus are not a long-stay population. The costs they quote exclude the costs for accommodation. Therefore, it is not surprising that community care costs less, although $43 \%$ of the cost might be considered high.

We costed the community care placements for a group of long-stay patients discharged into a 24 hour staffed hostel. The residential care for each was $£ 16722.66$ per year, compared with $£ 35000$ for a hospital bed. However, the items included in the hospital price need to be accounted for, such as mental health service input, psychology, occupational therapy and out-patient attendances. When these are added, the total cost comes to $£ 35880$ per resident per year. Drugs ( $£ 700$ per month for the 30 residents of the hostel, excluding depot medication) and primary care are excluded from the above figure.

In conclusion, we agree with Salize \& Rössler that it is vital accurately to cost community care but we wish to draw attention to the even greater importance of comparing like with like. Good, comprehensive community care costs more than hospital care; the most expensive patient in their cohort cost nearly half as much again as an in-patient.

SALIzE, H. J. \& RössleR, W. (1996) The cost of comprehensive care of people with schizophrenia living in the community. A cost evaluation from a German catchment area. British Journal of Psychiatry, 169, 42-48.

A. K. Stanley

Reaside Clinic

Birmingham B45 9BE

St Edwards Hospital

Cheddleton

Staffordshire ST13 7EB

J. F. MaCmillan
R. J. Craig

\section{Cytochromes and psychotropic drug interactions}

SIR: The editorial on cytochromes and psychotropic drug interactions (Taylor \& Lader, 1996) is timely. The recent advances in molecular biology and in the understanding of the cytochrome $\mathrm{p} 450$ system, in particular, need to be brought into the clinical arena. However, the implications of this work go beyond potential drug interactions.

The genotyping of the CYP2D locus has led to the exciting discovery not only of poor and extensive metabolisers but also of ultra-rapid metabolisers of the phenotypic probe, debrisoquine (Johansson et al, 1993). These show one or more copies of the CYP2D6 gene, the expression of which causes the hydroxylation of desbrisoquine (the first step in its breakdown). Since the oxidative metabolism and elimination of most phenothiazines is known to be mediated by the CYP2D6 isoenzyme, there is now a framework to understand patients who are treatment-resistant or who respond only to high levels of antipsychotic medication and appear unaffected by extrapyramidal or other side-effects (Bertilsson et al, 1993). Furthermore, recent studies have found an association between the poor debrisoquine metaboliser phenotype and increased likelihood of extrapyramidal side-effects (Arthur et al, 1995). It may be that once testing becomes more widely available outside specialist centres, genotyping of the CYP2D locus will become commonplace, providing predictive testing for concentrationdependent side-effects such as Parkinsonism, and informing clinical decisions on dosage of antipsychotic medication.

Arthur, H., DhHL, M. L., Stwers, B., et al (1995) Polymorphic drug metabolism in schizophrenic patients with tardive dyskinesia. Journal of Clinical Psychopharmacology, 15, 211-216.

Bertulsson, L., DAHL, M. L., SsoQvist, F., et al (1983) Molecular basis for rational megaprescriping in ultra rapid hydroxylators of debrisoquine. Lancet, 341, 63.

Johansson, I., Lundevist, E., Bertilsson, L., et al (1993) Inherited amplification of an active gene in the cytochrome P450 CYP2D locus as a cause of ultrarapid metabolism of debrisoquine. Proceedings of the National Academy of Sciences USA, $90,11825-11829$.

TAYLOR, D. \& LADER, M. (1996) Cytochromes and psychotropic drug interactions. British Journal of Psychiatry, 168, 529-532.

\section{Parkview Clinic}

G. D. L. BATES

60 Queensbridge Road

Moseley

Birmingham B13 8QE

Queen Elizabeth Psychiatric Hospital

Mindelsohn Way

Birmingham B15 2TZ 